\title{
Design of a Low-Cost Underwater Acoustic Modem
}

\author{
Bridget Benson, Ying Li, Brian Faunce, Kenneth Domond, Don Kimball, Curt Schurgers, and Ryan Kastner
}

\begin{abstract}
There has been an increasing interest in creating short-range, low data rate, underwater wireless sensor networks for scientific marine exploration and monitoring. However, the lack of an inexpensive, underwater acoustic modem is preventing the proliferation of these sensor networks. Thus, we are building an underwater acoustic modem starting with the most critical component from a cost perspective-the transducer. The design substitutes a commercial transducer with a homemade transducer using cheap piezoceramic material and builds the rest of the modem's components around the properties of the transducer to extract as much performance as possible. This letter describes the high level design, and cost and power characteristics of each of the major modem components: the transducer, the analog transceiver, and the digital signal processor of our modem prototype.
\end{abstract}

\section{INTRODUCTION}

$\mathbf{T}$ HERE is increasing interest in the design and deployment of underwater acoustic communication networks. For example, the persistent littoral undersea surveillance network (PLUSNet) demonstrates multisensor and multivehicle antisubmarine warfare (ASW) by means of an underwater acoustic communications network [1]. A short-range shallow water network to monitor pollution indicators in Newport Bay, CA, is proposed in [2]. A network of acoustic modems akin to motes is proposed for low power, short-range acoustic communications for seismic monitoring [3]. A swarm of acoustically networked autonomous drifters is envisioned to monitor phenomena as they are subjected to ocean currents [4]. The proposed underwater communication networks need access to tens to hundreds of inexpensive, low-power underwater acoustic modems, but existing commercial modems are too expensive and power consuming to be practical for these applications [5]. For example, Linkquest, Benthos, and DSPComm underwater modems all

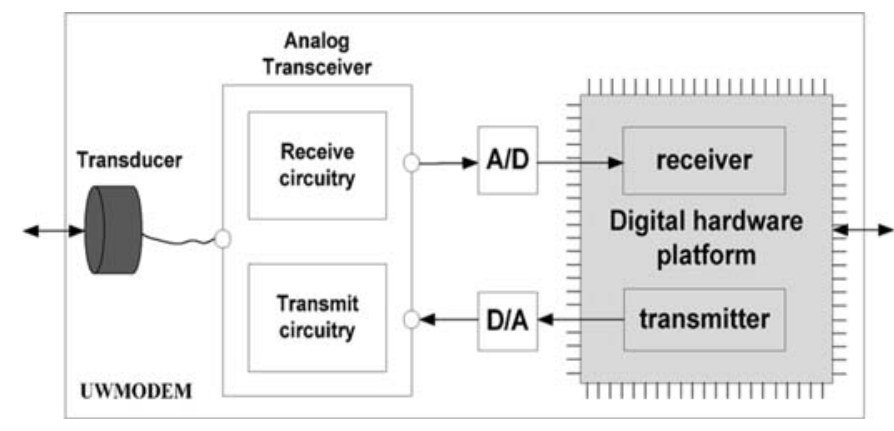

Fig. 1. Major components of an underwater acoustic modem.

cost more than $\$ 8000$ dollars [6]-[8], and require a minimum of $4 \mathrm{~W}$ transmit power. In order to make more short-range underwater acoustic communication networks a reality, the cost and power consumption of underwater acoustic modems must come down.

As shown in Fig. 1, underwater acoustic modems consist of three main components: an underwater transducer, an analog transceiver, and a digital hardware platform for control and signal processing. The most costly component is the underwater transducer as commercially available underwater omnidirectional transducers (such as those seen in existing research modem designs [9]-[11]) cost from $\$ 2000$ to $\$ 3000$. Therefore, much of the design for a low-cost modem lies in finding an appropriate substitute for the custom commercial transducer. Jurdak et al. substituted the transducer with generic, inexpensive speakers and microphones, but were only able to obtain a data rate of 42 bits per second (bps) for a transmission range of $17 \mathrm{~m}$ [12]. Benson et al. substituted a custom transducer with a commercially available fish finder transducer (which costs $\$ 50$ dollars), but were only able to obtain a data rate of $80 \mathrm{bps}$ for a transmission range of $6 \mathrm{~m} \mathrm{[13].}$

Thus, in this letter we present an overview of the design of an underwater acoustic modem starting with the most critical component from a cost perspective - the transducer. The design substitutes a commercial underwater transducer with a homemade underwater transducer using cheap piezoceramic material and builds the rest of the modem's components around the properties of the transducer to extract as much performance as possible. The goal of the design is to provide a low-cost, underwater acoustic modem that will be suitable for short-range sensor network applications.

The rest of this letter describes the high level design, cost and power characteristics of each of the major modem components: the transducer (Section II), the analog transceiver (Section III), and the digital signal processor (DSP) (Section IV). We conclude with a summary of the cost and power characteristics of the full design and a discussion of future work in Section V. 


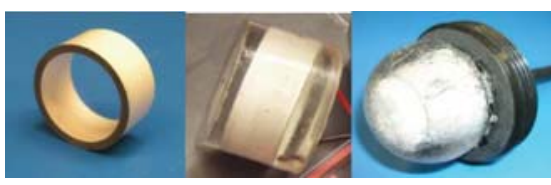

Fig. 2. From left to right: The raw piezoelectric ring ceramic, the potted ceramic, and the transducer in the potting compound mounted to a prototype plate to be attached to a modem housing.

\section{Transducer Impedance}

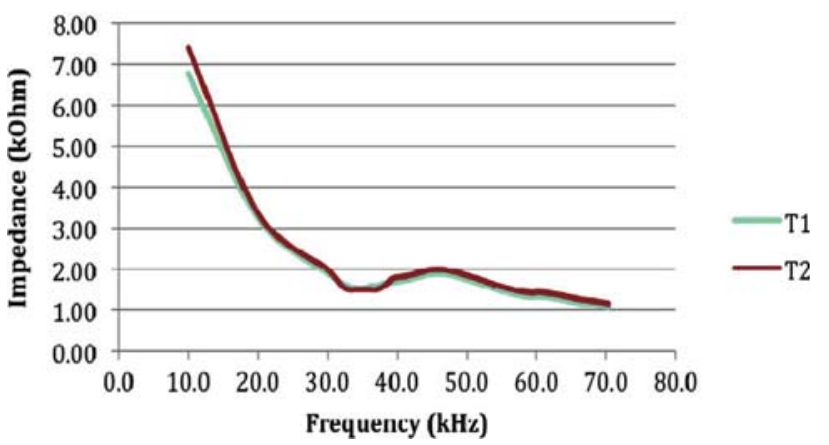

Fig. 3. Two transducers' (T1, T2) impedance at various frequencies. The resonant frequency occurs where the impedance first reaches a minimum.

\section{TRANSDUCER}

Underwater transducers are typically made from piezoelectric materials - materials (notably crystals such as lead zirconate titanate and certain ceramics) that generate an electric potential in response to applied mechanic stress and produce a stress or strain when an electric field is applied. The piezoelectric transducer needs to be encapsulated in a potting compound to prevent contact with any conductive fluids (Fig. 2). Commercial transducers are expensive, on the order of thousands of dollars, due to timeconsuming characterization and the subsequent optimization of the analog circuitry, which is further exacerbated by low volume production. However, the raw piezoelectric materials themselves are not very expensive and alternative cheaper, less accurate ways of characterizing and manufacturing the transducers exist. The key to our low-cost design is to forego a commercial underwater transducer, and use a homemade one built from low-cost piezoelectric material. The rest of the system is built around the properties of this inexpensive transducer to extract as much performance as possible.

Underwater transducers are typically omnidirectional in the horizontal plane and do not transmit in the vertical plane to reduce reflections off the surface and bottom [14]. This is especially important for shallow water communications. Our transducer uses a radially expanding ring that provides 2-D omnidirectionality in the plane perpendicular to the axis. Our transducer makes use of a \$10 dollar Steminc model SMC26D22H13 SMQA lead zirconate titanate (PZT) ceramic ring and a two-part urethane potting compound manufactured by Cytec Industries (Fig. 2). This particular potting compound was chosen because it has a density identical to that of water, which provides for efficient mechanical to acoustical energy coupling the water. Experimental tests have shown the ceramic maintains a linear output response up to $1000 \mathrm{~V}$ and $50 \mathrm{~W}$. Frequency response tests of two of our transducers (Fig. 3) indicate that the resonant

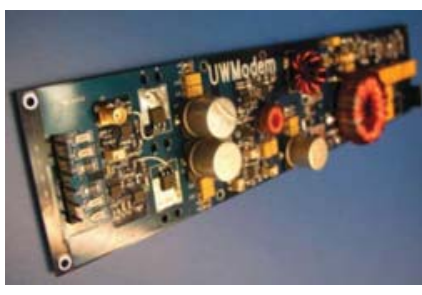

Fig. 4. Analog transceiver.

frequency of the transducer is $35 \mathrm{kHz}$ with a $\sim 6 \mathrm{kHz}$ bandwidth. Calibration tests giving the transducer's transmitting voltage response (TVR) and receiving voltage response (RVR) indicate it produces $140 \mathrm{~dB}$ re $\mathrm{uPa} / \mathrm{V} / \mathrm{m}$ at $35 \mathrm{kHz}$ and receives $-195 \mathrm{~dB}$ $\mathrm{re} \mathrm{V/1} \mathrm{uPa}$. When produced in large quantities, the estimated cost of the entire transducer including ceramic, potting, and labor is less than $\$ 50$ dollars.

\section{ANALOG TRANSCEIVER}

The analog transceiver (Fig. 4) consists of a high power transmitter and a highly sensitive receiver both of which are optimized to operate in the transducer's resonant frequency range. When produced in large quantities, the estimated cost of the transceiver is $\$ 125$ dollars. The transmitter and receiver portions of the analog transceiver are described in more detail in the following subsections.

\section{A. Transmitter}

The transmitter was designed to operate for signal inputs in a range from 0 to $100 \mathrm{kHz}$. The architecture is unique as it consists of two different amplifiers working in tandem. The primary amplifier is a highly linear Class $\mathrm{AB}$ amplifier that provides a voltage gain of 23 while achieving a power efficiency of about $50 \%$. The output of the Class AB amplifier is connected to current sense circuitry that in turn controls the secondary amplifier, which is a Class D switching amplifier. The Class D amplifier is inherently nonlinear, but possesses an efficiency of approximately $95 \%$. With both of the amplifiers driving the load and working together in parallel, the transmitter achieves a highly linear output signal while maintaining a power efficiency greater than $75 \%$. Because of its high linearity, the transmitter may be used with any modulation technique that can be programmed into the digital hardware platform.

A power management circuit is provided to adjust the output power in real-time to match it to the actual distance between transmitter and receiver. The ability to provide a low-power output has several important benefits: less interference for nearby ongoing communications; reduced noise pollution; and considerable power savings. The current configuration of the transmitter is equipped with a power management system that can switch between output levels of 2,12, 24, and $40 \mathrm{~W}$, which is efficiently coupled into the transducer in over its operating frequencies. The power management system has been designed so that the transmitter will maintain maximum efficiency over this wide range of power output levels. The system is controlled by a low-current $5 \mathrm{~V}$ signal from the digital hardware platform so that the power may be dynamically controlled for different operating conditions. 


\section{B. Receiver}

The receiver's architecture consists of a set of narrow (high-Q) filters with high gain. These filters are based on biquad band-pass filters, and essentially combine the tasks of filtering and amplification. The receiver is configured so that it only amplifies signals around $35 \mathrm{kHz}$ (to match the resonant frequency of the transducer), while attenuating low frequencies at a rate of $120 \mathrm{~dB}$ per decade and high frequencies at rate of 80 $\mathrm{dB}$ per decade. The receiver must be able to amplify only the frequencies of interest because of the large amount of noise associated with underwater acoustic signals. The current receiver configuration consumes about $375 \mathrm{~mW}$ when in standby mode and less than $750 \mathrm{~mW}$ when fully engaged. The relatively high power consumption [in comparison to that of the Woods Hole Oceanographic Institute (WHOI) Micromodem (200 mW)] is a result of the receiver's high gain $(65 \mathrm{~dB})$ which is capable of sufficiently amplifying an input signal as small as $10 \mathrm{mcV}$ allowing the receiver to pick up signals at longer distances. An ultralow-power wake up circuit will be added to the receiver to considerably reduce power consumption. A few receiver component values can be changed to widen its bandwidth (but decrease its gain) to allow for transmission of modulation schemes that require more bandwidth.

\section{Digital Signal Processor}

Our digital hardware platform makes use of reconfigurable hardware, particularly a field-programmable gate array (FPGA), for all the digital signal processing and control required for the modem. Reconfigurable devices provide a suitable digital hardware platform for the low-cost, low-power underwater acoustic modem as they strike a balance between solely hardware and solely software solutions. They have the programmability and nonrecurring engineering costs of software with performance capacity and energy efficiency approaching that of a custom hardware implementation [17]. Reconfigurable systems are known to provide the performance needed to process complex digital signal processing applications and especially provide increased performance benefits for highly parallel algorithms [15]-[18]. Furthermore, they are programmable allowing the same device to be used to implement a variety of different communication protocols. Unfortunately, they require substantially longer design time than a DSP.

We selected to implement frequency shift keying as our first physical layer communication protocol as it is a simple, but robust modulation scheme that requires a small bandwidth (to match the characteristics of our transducer and analog circuitry), and has been widely used in underwater communications over the past two decades due to its resistance to time and frequency spreading of the underwater acoustic channel [9], [19]. Our design platform is flexible and can be used to accommodate other modulation schemes in the future.

Table I shows the modem's time and frequency parameters. The "mark" frequency represents the frequency used to represent a digital " 1 " when converted to baseband and the "space" frequency represents the frequency used to represent a digital " 0 " when converted to baseband. The sampling frequency is used for sending and receiving the modulated waveform on the carrier frequency while the baseband frequency is used for all
TABLE I

FSK MODEM PARAMETERS

\begin{tabular}{|l|l|}
\hline Properties & Assignment \\
\hline Modulation & FSK \\
\hline Carrier frequency & $35 \mathrm{kHz}$ \\
\hline Mark frequency & $1 \mathrm{kHz}$ \\
\hline Space frequency & $2 \mathrm{kHz}$ \\
\hline Symbol duration & $5 \mathrm{~ms}$ \\
\hline Sampling Frequency & $192 \mathrm{kHz}$ \\
\hline Baseband Frequency & $16 \mathrm{kHz}$ \\
\hline
\end{tabular}

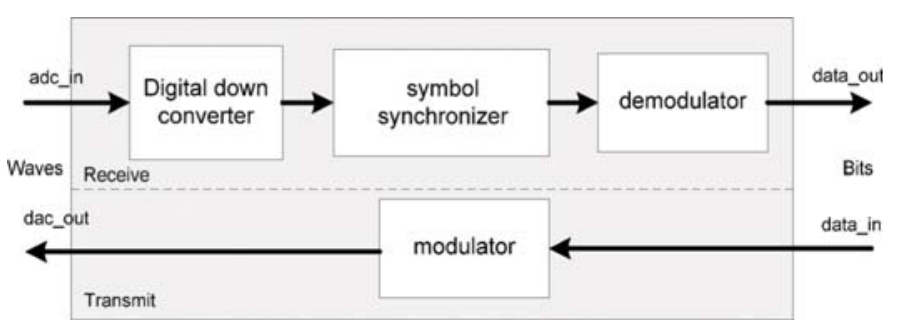

Fig. 5. Block diagram of a digital underwater acoustic modem.

TABLE II

FPGA RESOURCE USAGE

\begin{tabular}{|l|c|c|c|}
\hline & $\begin{array}{c}\text { Occupied } \\
\text { slices }\end{array}$ & LUTs & BRAMs \\
\hline Modulator & 95 & 184 & 9 \\
\hline DDC & 284 & 541 & 9 \\
\hline Demodulator & 1025 & 1980 & 1 \\
\hline Synchronizer & 12000 & 22101 & 2 \\
\hline
\end{tabular}

baseband processing. These frequencies were selected to provide sufficient oversampling of the desired frequency component while being integer multiples of one another.

Fig. 5. illustrates a block diagram of our FPGA implementation of an FSK modem. The most complicated block of the modem is the symbol synchronizer which is used to locate the correct start of the data sequence to set accurate sampling and decision timing for subsequent demodulation. The synchronizer is based on correlation with a known reference sequence (a 15-bit Gold code translated to an FSK waveform where a " -1 " is represented with the space frequency and a " 1 " is represented with the mark frequency). When the reference and receiving sequence exactly align with each other, the correlation result reaches a maximum value and the synchronization point is located. Details of the symbol synchronizer's implementation can be found in [20].

The design was implemented and successfully tested on a DINI Group DNMEG AD-DA evaluation board [21]. Hardware simulation results with packet lengths of 10000 symbols achieved a bit error rate of $10^{-2}$ at $10 \mathrm{~dB}$ SNR. Transmitting at $35 \mathrm{kHz}$, given a transmit source level of $170 \mathrm{~dB}$ re $1 \mathrm{uPa}$ and a noise level of $50 \mathrm{~dB}$ re $1 \mathrm{uPa}$, using the passive sonar equation with no directivity index, the signal may obtain $10 \mathrm{~dB}$ SNR at $1140 \mathrm{~m}$. Packets of 100 symbols were sent underwater in a 12-inch plastic bucket and achieved $0 \%$ bit error rate.

Table II shows the FPGA hardware resources required for the components of the acoustic modem design with standard optimization. These resources are reported for a Xilinx Spartan 3 xc3s4000 FPGA. The power consumption, determined by XPower Estimator 9.1.03, is $0.379 \mathrm{~W}$. The design offers a 
TABLE III

ESTIMATED LARGE VOLUME COSTS

\begin{tabular}{|l|l|}
\hline & Cost $\mathbf{( \$ )}$ \\
\hline Transducer & 50 \\
\hline Transceiver & 125 \\
\hline Digital Components & 75 \\
\hline Batteries & 100 \\
\hline Housing & 150 \\
\hline Power Supply & 100 \\
\hline Total & $\mathbf{6 0 0}$ \\
\hline
\end{tabular}

TABLE IV

MODEM POWER CHARACTERISTICS

\begin{tabular}{|l|l|}
\hline & Power $(\mathbf{W})$ \\
\hline Analog Transmitter & $2,12,24,40$ \\
\hline Analog Receiver & 0.75 \\
\hline Digital Modem & 0.379 \\
\hline ADC & TBD \\
\hline DAC & TBD \\
\hline
\end{tabular}

compact implementation capable of fitting into a low-power device that costs less than $\$ 70$ dollars.

\section{CONCLUSION}

We have described the major design components of our prototype low-cost, underwater acoustic modem intended for shortrange, low data rate sensor network applications. Currently, the digital hardware platform has been tested and supports data rates of up to 200 bps with $10^{-2}$ bit error rate at a low signal to noise ratio $(\mathrm{SNR})$ of $(10 \mathrm{~dB})$. Initial in-water tests of the transducer and analog transceiver in Mission Bay, CA, indicate our design is capable of detecting a signal at distances greater than $350 \mathrm{~m}$. We are currently developing a power supply board, battery pack, and watertight housing (that can withstand pressures at depth of up to $100 \mathrm{~m}$ ) to be able to test the modem in the open ocean in order to assess its performance. Table III provides cost estimates for a fully packaged underwater modem and Table IV summarizes its current power consumption.

The power consumption of the analog-to-digital converter (ADC) and digital-to-analog converter (DAC) are specified to be determined (TBD) as the devices on our evaluation board are over specified and too power consuming for our low-power design. In the future, to further reduce power consumption, we plan to explore the possibilities to provide signal detection at even lower power levels. This is paramount to building a modem that has low listening power, which is also a key requirement to ensure long lifetime on a limited battery supply. We plan to eventually utilize a design that has a programmable gain, which is dynamically controlled by the digital hardware platform. In addition, further changes to the circuit design of the transceiver will be made to further increase its efficiency.

\section{REFERENCES}

[1] M. Grund, L. Freitag, J. Preisig, and K. Ball, "The PLUSNet underwater communications system: Acoustic telemetry for undersea surveillance," in Proc. IEEE Oceans Conf., Boston, MA, Sep. 2006.

[2] R. Jurdak, C. V. Lopes, and P. Baldi, "Battery lifetime estimation and optimization for underwater sensor networks," in Sensor Network Operations. New York: Wiley, May 2006, pp. 397-420.

[3] J. Wills, W. Ye, and J. Heidemann, "Low-power acoustic modem for dense underwater sensor networks," in Proc. ACM Int. Workshop Underwater Netw., Los Angeles, CA, 2006.

[4] J. Heidemann, Y. Li, A. Syed, J. Wills, and W. Ye, "Research challenges and applications for underwater sensor networking," in Proc. IEEE Wireless Commun. Netw. Conf., New Orleans, LA, 2006.

[5] J. Jaffe and C. Schurgers, "Sensor networks of freely drifting autonomous underwater explorers," in Proc. ACM Int. Workshop Underwater Netw., Los Angeles, CA, 2006.

[6] Benthos, Inc. Underwater Acoustic Modems [Online]. Available: http://www.benthos.com/pdf/Modems/ModemBrochure.pdf

[7] LinkQuest, Inc. Underwater Acoustic Modems [Online]. Available: http://www.link-quest.com/html/uwm_hr.pdf

[8] DSPCOMM, Underwater Wireless Modem [Online]. Available: http:// www.dspcomm.com

[9] L. Freitag, M. Grund, S. Singh, J. Partan, P. Koski, and K. Ball, "The WHOI micro-modem: An acoustic communications and navigation system for multiple platforms," in Proc. IEEE OCEANS Conf., Washington DC, Sep. 2005.

[10] R. A. Iltis, H. Lee, R. Kastner, D. Doonan, T. Fu, R. Moore, and M. Chin, "An underwater acoustic telemetry modem for eco-sensing," in Proc. IEEE OCEANS Conf., Washington DC, Sep. 2005.

[11] H. Yan, S. Zhou, Z. Shi, and B. Li, "A DSP implementation of OFDM acoustic modem," in Proc. ACM Int. Workshop Underwater Netw., Montreal, QC, Canada, 2007.

[12] R. Jurdak, P. Aguiar, P. Baldi, and C. V. Lopes, "Software modems for underwater sensor networks," in Proc. IEEE OCEANS Conf., Washington DC, Sep. 2005.

[13] B. Benson, G. Chang, D. Manov, B. Graham, and R. Kastner, "Design of a low-cost acoustic modem for moored oceanographic applications," in Proc. ACM Int. Workshop Underwater Netw., Los Angeles, CA, 2006.

[14] C. H. Sherman and J. L. Butler, Transducers and Arrays for Underwater Sound. New York: Springer-Verlag, 2007.

[15] K. Bondalapati and V. K. Prasanna, "Reconfigurable computing systems," Proc. IEEE, vol. 90, no. 7, pp. 1201-1217, Jul. 2007.

[16] K. Compton and S. Hauck, "Reconfigurable computing: A survey of systems and software," ACM Comput. Surveys, vol. 34, pp. 171-210, 2002.

[17] R. Kastner, A. Kaplan, and M. Sarrafzadeh, Synthesis Techniques and Optimizations for Reconfigurable Systems. Boston, MA: Kluwer, 2004.

[18] B. Benson, A. Irturk, J. Cho, and R. Kastner, "Energy benefits of reconfigurable hardware for use in underwater sensor nets," in Proc. IEEE Reconfigurable Arch. Workshop, Rome, Italy, May 2009.

[19] D. B. Kilfoyle and A. B. Baggeroer, "The state of the art in underwater acoustic telemetry," IEEE J. Oceanic Eng., vol. 25, no. 1, pp. 4-27, Jan. 2000.

[20] Y. Li, B. Benson, X. Zhang, and R. Kastner, "Hardware implementation of symbol synchronization for underwater FSK," in Proc. IEEE Int. Conf. Sensor Netw., Ubiquitous, Trustworthy Comput., Newport Beach, CA, 2010

[21] DINI Group [Online]. Available: http://www.dinigroup.com/index. php?product=DNMEG_ADDA 